\title{
Time-frequency Characterization of Simultaneous Intra-atrial and Electrocardiographic Recordings During Atrial Fibrillation
}

\author{
M Stridh ${ }^{1}$, A Bollmann ${ }^{2,3}$, D Husser ${ }^{2,3}$, A. K. Bhandari ${ }^{2}$ D. S. Cannom ${ }^{2}$, L Sörnmo ${ }^{1}$ \\ ${ }^{1}$ Dept. of Electroscience, Lund University, Sweden, ${ }^{2}$ Departments of Cardiology, Good Samaritan \\ Hospital and Harbor-UCLA Medical Center, Los Angeles, CA, USA, ${ }^{3}$ Dept. of Cardiology, \\ Otto-von-Guericke-University, Magdeburg, Germany
}

\begin{abstract}
A new method for characterization of simultaneous intraatrial and ECG recordings during atrial fibrillation $(A F)$ is presented. With different preprocessing, both types of signals are characterized using a logarithmic timefrequency distribution from which trends of frequency, regularity, morphology (only ECG) and signal quality are extracted. The objective of the study is to relate ECG measures of rate and morphology to measures of intra-atrial organisation. The algorithm has been tested on a database with simultaneous ECG and right atrial recordings from 34 patients with drug-refractory $A F$. The average frequency in lead $V_{1}$ was $5.91 \mathrm{~Hz}($ std=0.94) and the average exponential decay of harmonic magnitudes (reflecting the morphology) in $V_{1}$ was 1.31 (std=0.38); their correlation coefficient was $0.53(p<0.001)$. The correlation coefficient between spatial frequency dispersion in the right atrium and harmonic decay was 0.53 ( $p<0.005)$. More organized $A F$, observed in patients treated with amiodarone, was reflected by a lower frequency, smaller right atrial frequency dispersion and lower exponential decay.
\end{abstract}

\section{Introduction}

Characterization of AF using the ECG is typically performed by first extracting the atrial component from the ECG [1]. Frequency analysis of the residual ECG after extraction of atrial activity has been suggested by several research groups for the purpose of estimating the dominant rate of the arrhythmia [2], [3]. During the last years, a new generation of methods for atrial signal characterization has evolved with the ability to follow the dynamics of atrial signal properties, allowing more advanced studies of mechanisms and treatment strategies [4], [5], [6].

Electrogram-based characterization of AF has been mainly focused on atrial organization by measuring the repetitiveness of consecutive activation [7], spatial crosscorrelation decay [8], or by using methods based on nonlinear dynamics [9]. Frequency analysis has also been used to measure activation rates in different parts of the atria [10],[11].

Recently, we have developed a method for timefrequency characterization of atrial tachyarrhythmias in the surface ECG. In the present paper, this method is extended to perform analysis of intra-atrial electrograms for the purpose of investigating and relating signal properties of the ECG and intra-atrial electrograms. The database used in this study is described in Sec. 2, and the method and results are presented in Secs. 3 and 4, respectively. Finally, the outcome of the present study is discussed in Sec. 5 .

\section{Database}

This study includes 36 patients with drug-refractory AF who underwent pulmonary vein (PV) catheter ablation. Electrophysiologic atrial studies were performed using catheters positioned in the right atrial wall (one electrogram, $n=36$ and five adjacent electrograms, $n=27$ ), the coronary sinus $(n=28)$ and the pulmonary veins $(n=28)$. A subgroup of the patients was treated with amiodarone $(n=7)$. Before proceeding with the ablation procedure, 12-lead ECG and endocardial electrograms were recorded and stored using a commercial recording system (Bard EP Lab System Duo, Billerica, USA). All recordings were sampled with $1 \mathrm{kHz}$ and were of 10 seconds duration. A recording example with three ECG leads, following QRST cancellation, and five closely spaced right atrial electrograms is shown in Fig. 1.

\section{Methods}

In this work, our sequential atrial signal characterization method, [4], was used to analyze both ECG and intraatrial recordings. A brief description of the method along with a description of the different preprocessing strategies required for the two signal types are presented below. 


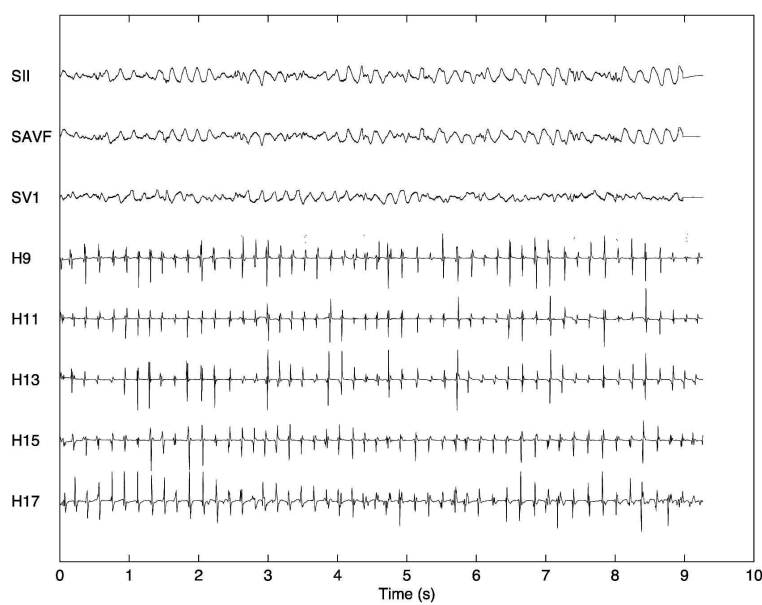

Figure 1. A signal example with three ECG leads after QRST cancellation followed by five closely spaced right atrial electrograms.

\section{Time-frequency characterization}

The sequential atrial signal characterization, originally developed for ECG analysis, is based on time-frequency analysis using a logarithmic frequency scale. The method is sequential in that it starts with a simple spectral profile constructed as a zero spectrum with a peak at a certain predefined frequency and then sequentially matches this spectral profile to each new time slice of the time-frequency distribution. The matching leads to robust estimation of the frequency and amplitude taking the entire spectrum into account in the estimation procedure. Once the estimates are determined the current spectrum is aligned and weighted into the spectral profile. In this way, the spectral profile will adapt to the signal and the estimation will become more and more robust as the spectral profile better represents the signal structure/morphology. For short signals, such as those used in this study, the spectral profile is instead initiated as the conventional spectrum of the signal and the adaptation rate is increased in order to converge faster. Alignment of two spectra which both have harmonic structures motivates the need for the logarithmic frequency scale.

The exponential decay of the harmonic magnitudes in the spectral profile is used as a coarse measure of waveform morphology. Large harmonics reflect a signal with sharp edges (short duration) compared to its period. Atrial tachycardia with isoelectric baseline (and $\mathrm{P}$ waves), with the main part of the energy in a small part of the period, typically has large harmonics, and thus a low exponential decay of the harmonic magnitudes. Medium harmonic magnitudes correspond to a signal whose energy is more evenly spread in time over the period, but still with relatively sharp edges. The "sawtooth-like" signals without isoelectric baseline have medium harmonic magnitudes and medium exponential decay. Finally, signals with low or no harmonics corresponds to a fluctuating signal without sharp edges. Thus, the decay of the harmonic magnitudes may be interpreted as a coarse measure of atrial organization; a tachycardia or sawtooth-like signal correspond to a more organized activation patterns compared to a more randomly fluctuating atrial signal.

The sequential characterization method uses overlapping windows of short duration (2.56 s long, 1 window per second) in order to provide trends of the different signal parameters. The method requires that the atrial signal is relatively clean from disturbances, its spectral content is below $25 \mathrm{~Hz}$, and the harmonic pattern to only contain magnitudes lower than that of the fundamental frequency (which is typically the case for, e.g., lead $V_{1}$ ).

Two measures are extracted from the ECG $\left(\mathrm{V}_{1}\right)$, namely average (temporal) frequency and exponential decay. From the intra-atrial signals, several measures are calculated: temporal frequency averages from each electrogram are spatially averaged into one frequency estimate for each part of the atria, i.e., for the right atrium, coronary sinus and pulmonary veins, respectively. For the recordings with five adjacent electrograms in the right atrium, the frequency dispersion (max-min), is also calculated and was based on five adjacent electrograms from anywhere on the catheter.

\section{Preprocessing}

The preprocessing stages needed to analyze the surface ECG signals are: pacemaker detection/suppression, beat detection/classification, QRST cancellation, quality control and decimation to $50 \mathrm{~Hz}$. The intra-atrial signals have a completely different structure and requires different preprocessing. In order to make the intra-atrial signals suitable for the signal characterization method, a rectifier, an amplitude compressor and a lowpass filter is used instead. With this approach, the resulting signal has spectral characteristics similar to those of the atrial component of the ECG. At some atrial sites, the signal is still sharper than is typically the case in the body surface leads which may result in that the first harmonic is slightly larger than the fundamental. Therefore, the characterization algorithm for intra-atrial signals is modified such that all spectra are weighted with a linearly decreasing function before being compared with and introduced into the spectral profile. In this way, signals with sharper waveforms can be handled by the algorithm. Pacemaker suppression is, if needed, also performed for the intra-atrial signals although the detection is based only on the ECG leads. An overview of the preprocessing strategies is shown in Fig. 2. In addition to the above processing, baseline filtering and powerline 


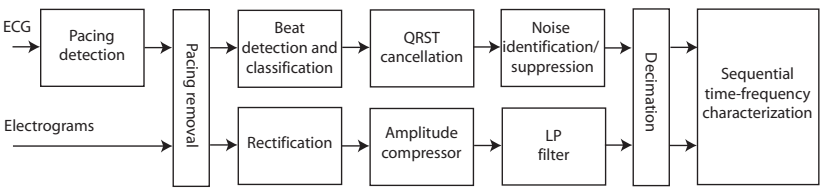

Figure 2. Preprocessing strategies for surface ECG and intra-atrial electrograms.

interference reduction was used for both types of signals. An example of an analyzed signal is shown in Fig. 3.
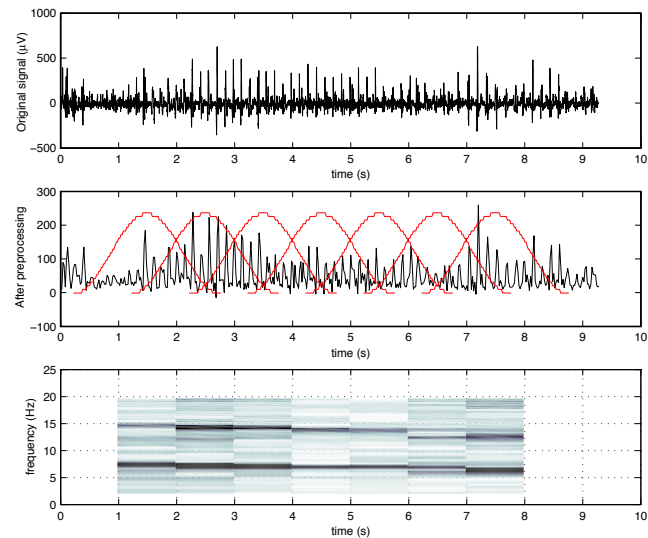

Figure 3. Analysis example: (a) The original intra-atrial signal, (b) the preprocessed signal plotted with analysis windows, and (c) the resulting time-frequency distribution showing a repetition rate just below $7 \mathrm{~Hz}$.

\section{Results}

For the entire database, the average frequency in lead $\mathrm{V}_{1}$ was $5.91 \mathrm{~Hz}(\mathrm{std}=0.94 \mathrm{~Hz})$ and the overall average exponential decay was $1.31 \quad(\mathrm{std}=0.38)$. The invasively measured right atrial frequency dispersion was, based on recordings with multiple adjacent right atrial electrograms, in average $0.46 \mathrm{~Hz}$. The correlation between the frequency $\left(\mathrm{V}_{1}\right)$ and exponential decay $\left(\mathrm{V}_{1}\right)$ was $0.53(\mathrm{p}<0.001, \mathrm{n}=36)$ and the correlation between the average frequency in lead $\mathrm{V}_{1}$ and the average (temporal and spatial) of the right atrial frequency was $0.81(\mathrm{p}<<0.001, \mathrm{n}=36)$. In fact, the frequency in lead $V_{1}$ was slightly better correlated with the maximum frequency measured in the right atrium $(0.94$, $\mathrm{p}<<0.001)$ than with the spatially averaged frequency. See Figs. 4 (a) and (b) for the corresponding patient distributions. The correlation between the exponential decay $\left(\mathrm{V}_{1}\right)$ and the right atrial frequency dispersion was $0.53(\mathrm{p}<0.005, \mathrm{n}=27)$ thus relating ECG morphology with
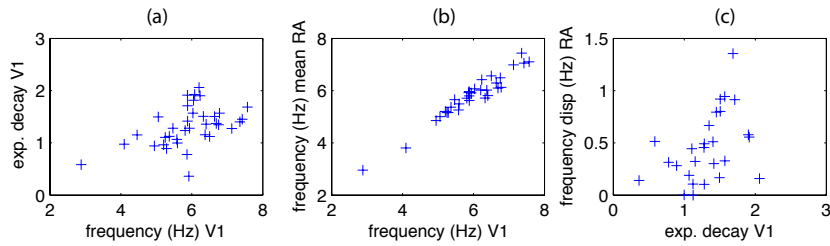

Figure 4. Distribution of measures plotted as (a) frequency $\left(\mathrm{V}_{1}\right)$ versus exponential decay, (b) frequency $\left(\mathrm{V}_{1}\right)$ versus frequency (right atrium), and (c) right atrial frequency dispersion versus exponential decay.

right atrial organization, see Fig. 4(c). An illustration of the relation between the ECG waveform and the right atrial organisation is shown in Fig. 5 where two segments with only a few seconds in between are shown. Note that the activities in other parts of the atria or pulmonary veins do not change in this particular case.

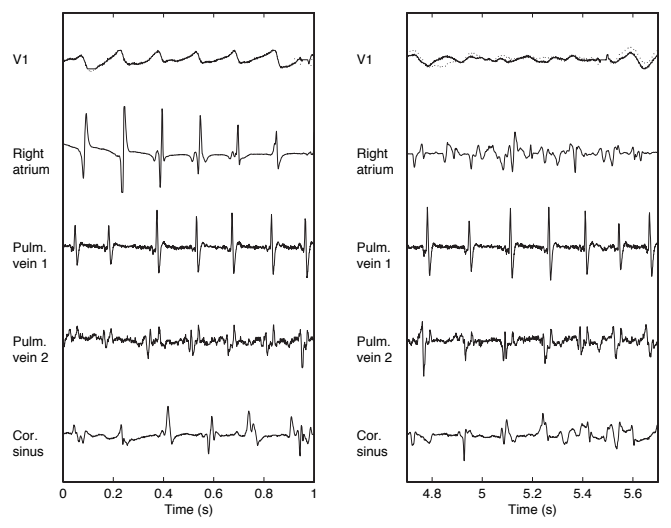

Figure 5. Simultaneously measured ECG $\left(\mathrm{V}_{1}\right)$ and intraatrial electrograms. Right panel is measured a few second later compared to the left panel.

For the subgroup of patients on amiodarone, the average frequency was $4.76 \mathrm{~Hz}(\mathrm{std}=1.06 \mathrm{~Hz})$ and for those not treated with amiodarone $6.19 \mathrm{~Hz}(\operatorname{std}=0.66 \mathrm{~Hz})$; the difference was significant with $\mathrm{p}<<0.001$. Similarly, the exponential decay was in average $1.06(\mathrm{std}=0.29)$ for the amiodarone patients and $1.38(\mathrm{std}=0.38)$ for the others $(\mathrm{p}<0.05)$. These two measures indicate a more organized ECG pattern with slower rate and more distinct f-waves for the amiodarone patient compared to the others. When comparing with the invasively measured right atrial frequency dispersion, a similar pattern can be seen with a frequency dispersion of $0.27 \mathrm{~Hz}$ in the amiodarone patients and $0.50 \mathrm{~Hz}$ in those not on amiodarone. The distributions of lead $\mathrm{V}_{1}$ frequency, exponential decay and right atrial frequency dispersion for patients with and 
without amiodarone is shown in Fig. 6 and are summarized in Table 1.

\begin{tabular}{|c|c|c|c|}
\hline Patients & freq. (std) $\mathrm{V}_{1} / \mathrm{Hz}$ & exp. dec. (std) $\mathrm{V}_{1}$ & freq. disp RA/Hz \\
\hline All & $5.91(0.94) \mathrm{Hz}$ & $1.31(0.38)$ & 0.46 \\
\hline With amio. & $4.76(1.06)$ & $1.06(0.29)$ & 0.27 \\
\hline Without amio. & $6.19(0.66)$ & $1.38(0.38)$ & 0.50 \\
\hline
\end{tabular}

Table 1. Summary of the effect of amiodarone.
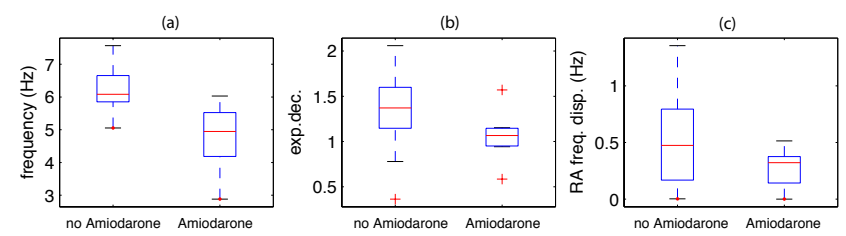

Figure 6. Distribution of (a) frequency, (b) exponential decay, and (c) right atrial frequency dispersion. For (a) and (b) there were 29 without and 7 with amiodarone. The corresponding values for (c) were 22/5.

\section{Discussion}

This study has explored the pathophysiologic meaning of the ECG waveform expressed as the exponential decay by comparing it with invasively measured right atrial frequency characteristics. The exponential decay was correlated with right atrial frequency dispersion indicating that it may be used as a measure of AF organization. This is supported by the fact that patients treated with amiodarone, known to increase AF organisation, had lower exponential decay values as well as lower right atrial frequency dispersion [12]. There was also a strong correlation between exponential decay and frequency in lead $V_{1}$ (which is very well-correlated with intra-atrial frequency) which is in accordance with previous invasive studies showing that more organized AF has lower frequency [13].

\section{Acknowledgements}

Dr. Bollmann was supported by the Max Kade Foundation, Inc., New York, NY, USA. Dr. Husser was supported by a NBL-3 travel grant from the University Hospital Magdeburg, Germany. This study has been performed in part within the NordForsk network "Electrocardiology in Atrial Fibrillation".

\section{References}

[1] Langley P, Stridh M, Rieta JJ, Millet-Roig J, Sörnmo L, Murray A. Reconstruction of atrial signals derived from the 12-lead ecg using atrial signal extraction techniques. In Proc. Comput. in Cardiol. IEEE Press, 2003; 129-132.

[2] Holm M, Pehrsson S, Ingemansson M, Sörnmo L, Johansson R, Sandhall L, Sunemark M, Smideberg B, Olsson C, Olsson SB. Non-invasive assessment of atrial refractoriness during atrial fibrillation in man-introducing, validating and illustrating a new ECG method. Cardiovasc Res 1998;38:6981.

[3] Bollmann A, Kanuru N, McTeague K, Walter P, DeLurgio D, Langberg J. Frequency analysis of human atrial fibrillation using the surface electrocardiogram and its response to ibutilide. Am J Card 1998;81:1439-1445.

[4] Stridh M, Sörnmo L, Meurling CJ, Olsson SB. Sequential characterization of atrial tachyarrhythmias based on ECG time-frequency analysis. IEEE Trans Biomed Eng 2004; 51(1):100-114.

[5] $\mathrm{Ng} \mathrm{J}$, Sahakian A, Swiryn S. Vector analysis of atrial activity from surface ECG's recorded during atrial fibrillation. In Proc. Comput. in Cardiol. IEEE Press, 2002; 21-24.

[6] Narayan S, Bhargava V. Temporal and spatial phase analysis of the electrocardiogram stratify intra-atrial and intra-ventricular organization. IEEE Trans Biomed Eng 2004;51(10):1749-1764.

[7] Faes L, Nollo G, Antolini R, Gaita F, Ravelli F. A method for quantifying atrial fibrillation organization based on wavemorphology similarity. IEEE Trans Biomed Eng 2002; 49:1504-1513.

[8] Botteron D, Smith J. A technique for measurement of the extent of spatial organization of atrial activation during atrial fibrillation in the intact human heart. IEEE Trans Biomed Eng 1995;42(6):579-586.

[9] Censi F, Barbaro V, Bartolini P, Calcagnini G, Michelucci A, Cerutti S. Non-linear coupling of atrial activation processes during atrial fibrillation in humans. Biol Cybern 2001; 85:195-201.

[10] Lazar S, Dixit S, Marchlinski FE, Callans D, Gerstenfeld EP. Presence of left-to-right atrial gradient in paroxysmal but not persistent atrial fibrillation. Circ 2004;110:3181-3186.

[11] Husser D, Stridh M, Sörnmo L, Cannom D, Bhandari A, Girskij M, Olsson S, Bollmann A. Relation of pulmonary venous fibrillatory rates to rates obtained from the surface electrocardiogram in persistent atrial fibrillation. In Proc. Comput. in Cardiol. IEEE Press, 2004; 241-244.

[12] Husser D, Stridh M, Sörnmo L, Geller C, Klein H, Olsson S, Bollmann A. Time-frequency analysis of the surface electrocardiogram for monitoring antiarrhythmic drug effects in atrial fibrillation. Am J Cardiol 2005; 95(4):526-528.

[13] Konings K, Kirchhof C, Smeets J, Wellens H, Penn O, Allessie M. High-density mapping of electrically induced atrial fibrillation in humans. Circ 1994;89:1665-1680.

Address for correspondence:

Martin Stridh (martin.stridh@es.lth.se)

Dept. of Electroscience, Lund University

Box 118, SE-221 00 Lund, SWEDEN 